\title{
Erratum: Compatibility in multiparameter quantum metrology [Phys. Rev. A 94, 052108 (2016)]
}

\author{
Sammy Ragy, Marcin Jarzyna, and Rafał Demkowicz-Dobrzański
}

(Q) (Received 30 January 2019; published 25 February 2019)

DOI: 10.1103/PhysRevA.99.029905

There is a small error in the expression of the Holevo Cramér-Rao bound in Eq. (6), which should be written as

$$
\operatorname{Tr}[G \operatorname{Cov}(\tilde{\boldsymbol{\varphi}})] \geqslant \min _{\left\{X_{i}\right\}}\left\{\operatorname{Tr}(G \operatorname{Re} V)+\|\sqrt{G} \operatorname{Im} V \sqrt{G}\|_{1}\right\}
$$

where the second term has been changed from $\|G \operatorname{Im} V\|_{1}$, and the various symbols are defined in the original text. This term and its replacement coincide when $G \operatorname{Im} V$ is a normal operator. In particular, this is true when $G$ and $\operatorname{Im} V$ commute, but not in general. The proofs and results of the paper are unchanged.

We thank S. Zhou and L. Jiang for spotting the error. 